\title{
A non-singular transformation whose spectrum has Lebesgue component of multiplicity one
}

\author{
e. H. el Abdalaoui and M. G. Nadkarni
}

\begin{abstract}
In this note we give an example of an ergodic non-singular map whose unitary operator admits a Lebesgue component of multiplicity one in its spectrum.
\end{abstract}

\section{Introduction}

The purpose of this paper is to give an example of an ergodic non-singular transformation whose associated unitary operator admits Lebesgue component of multiplicity one in its spectrum. M. Guenais [7] and Downarowicz-Lacroix 8 ] have shown that there exist measure preserving transformations with such property provided there exists a sequence of analytic trigonometric polynomials $P_{n}, n=1,2, \cdots$ whose absolute values $\left|P_{n}\right|, n=1,2, \cdots$ converge to 1 in some sense, and such that for each $n$, the coefficients of $P_{n}$ are real and equal in absolute value. In [7, the initiating paper, M. Guenais requires existence of such a sequence $P_{n}, n=1,2, \cdots$ whose $L^{1}$ norms converge to 1 . Downarowicz and Lacroix requires the existence of the so called Barker polynomials of arbitrary high length. If we dispense with the requirement that the transformations be measure preserving but require only that they be non-singular and ergodic then the problem has a non-speculative and concrete solution, for partial sums of power series expansions finite Blaschke products with real (non-vanishing) zeros provide us with ultraflat polynomials $P_{n}, n=1,2, \cdots$ with real coefficients, where, for any $n$ the coefficients of $P_{n}, n=1,2, \cdots$ are not equal in absolute value, but which can be used to construct non-singular ergodic transformations with the desired spectral property. We also mention that Guenais has constructed a concrete egodic measure preserving group action whose unitary

2010 Mathematics Subject Classification. Primary 37A05, 37A30, 37A40; Secondary 42A05, $42 \mathrm{~A} 55$.

Key words and phrases. simple Lebesgue spectrum, Banach problem, singular spectrum, non-singular maps, rank one maps, generalized Riesz products, flat polynomials, ultraflat polynomials, Littlewood problem. 
group admits Haar component of multiplicity one in its spectrum. Our result shows that in the non-singular category one can get integer action with this property. We refer the reader to our papers [1, 2] for a fuller discussion on connection between flat polynomials, $H^{p}$ theory and spectral questions in ergodic theory.

\section{Simple Blaschke Product and Ultraflat Sequence of Polynomials}

Let $S^{1}$ denote the circle group and $d z$ the normalized Lebesgue measure on $S^{1}$. A sequence $P_{n}(z), n=1,2, \cdots$ of analytic trigonometric polynomials of $L^{2}\left(S^{1}, d z\right)$ norm 1 is said to be ultraflat if the sequence $\left|P_{n}(z)\right|, n=1,2, \cdots$ converges uniformly to the constant function 1 as $n \rightarrow \infty$. J. E. Littlewood [15] asked if there exists an ultraflat sequence where, for each $n$, coefficients of $P_{n}$ are equal in absolute value, a question which J-P. Kahane 13 answered in the affirmative. The coefficients of polynomials in the ultraflat sequence constructed by Kahane are complex. It is an open question if there exist an ultraflat sequence of polynomials $P_{n}, n=1,2, \cdots$ where for each $n$, coefficients of $P_{n}$ are real and equal in absolute value.

Our purpose is served by an ultraflat sequence $P_{n}, n=1,2, \cdots$, with coefficients of $P_{n}$ real and bounded away from 1 in absolute value. The sequence of partial sums of power series of a finite Blaschke product with real (non-vanishing) zeros provides us with such ultraflat sequence. We will use only the simplest of such Blaschke products.

Consider the linear fractional map $\frac{z-\alpha}{1-\alpha z}$, where $\alpha$ is real positive and less than one. This function maps the unit circle onto the unit circle and analytic in the closed disk of radius $\frac{1}{\alpha}>1$, so has a power series expansion valid on $S^{1}$.

$$
\begin{aligned}
(z-\alpha) \sum_{j=0}^{\infty} \alpha^{j} z^{j} & =\sum_{j=0}^{\infty} \alpha^{j} z^{j+1}-\sum_{j=0}^{\infty} \alpha^{j+1} z^{j} \\
& =-\alpha+\sum_{j=1}^{\infty} \alpha^{j-1}\left(1-\alpha^{2}\right) z^{j}
\end{aligned}
$$

It can be checked that the sum of the squares of the coefficients of this power series is 1 , as it should be, since it is a function of absolute value 1 on $S^{1}$. The sequence of finite sums

$$
R_{n}(z)=-\alpha+\sum_{j=1}^{n-1} \alpha^{j-1}\left(1-\alpha^{2}\right) z^{j}, n=1,2, \cdots
$$

when divided by their $L^{2}\left(S^{1}, d z\right)$ norms, is therefore ultraflat. Since $\left\|R_{n}\right\|_{2} \rightarrow 1$ as $n \rightarrow \infty$, we see that for all $n \geq 2$, coefficients of $\frac{R_{n}}{\left\|R_{n}\right\|_{2}}$ in absolute value are less than some $\lambda<1$. 
Let $Q_{m}=\frac{R_{m}}{\left\|R_{m}\right\|_{2}}$ and choose a subsequence $Q_{m_{k}}, k=1,2, \cdots$ of $Q_{m}, m=$ $1,2, \cdots$ such that $\| Q_{m_{k}}(z)|-1|<\frac{1}{2^{k}}, k=1,2, \cdots$. Write $P_{k}=Q_{m_{k}}$. Clearly for any positive integer $l, \| P_{k}\left(z^{l}\right)|-1|<\frac{1}{2^{k}}$ and $P_{k}, k=1,2, \cdots$ are bounded away from zero on $S^{1}$. Let $h_{0}=1, h_{j}=m_{1} m_{2} \cdots m_{j}, j \geq 1$. We note that the finite products $\prod_{j=1}^{k}\left|P_{j}\left(z^{h_{j-1}}\right)\right|, k=1,2, \cdots$ converge uniformly to a non-vanishing function $g$ whose square is also non-vanishing continuous function on $S^{1}$. We claim that $\int_{S^{1}} g^{2} d z=1$. Note that the constant term of $\left|P_{j}\left(z^{h_{j}}\right)\right|^{2}$ is 1 . Further the highest power of $z$ in $P_{1}(z) P_{2}\left(z^{h_{1}}\right) \cdots P_{k}\left(z^{h_{k-1}}\right)$ is $h_{k}-1$, and the powers of $z$ in $P_{k+1}\left(z^{h_{k}}\right)$ are multiples of $h_{k}$ so the constant term of

$$
\prod_{j=1}^{k}\left|P_{j}\left(z^{h_{j-1}}\right)\right|^{2}
$$

is also 1 . So these products integrate to 1 . Clearly, since products (1) converges to $g^{2}$ uniformly, $\int_{S^{1}} g^{2} d z=1$

\section{Unitary operators $U_{T}$ and $V_{\phi}$}

We will now construct a non-singular rank one non-dissipative, ergodic transformation $T$ on the unit interval (equipped with Lebesgue measure $\nu$ ) and a function $\phi$ on $[0,1]$ taking values -1 and 1 such that the maximal spectral type of the unitary operator $V=V_{\phi}$ defined by

$$
\left(V_{\phi} f\right)(x)=\phi(x)\left(\frac{d \nu \circ T}{d \nu}(x)\right)^{\frac{1}{2}} f(T x), f \in L^{2}([0,1], \nu)
$$

has simple spectrum with maximal spectral type $g^{2} d z$. The transformation $T$ will be a generalized odometer.

\section{Ultraflat Polynomials $P_{k}, k=1,2, \cdots$ and rank one transformation}

Let

$$
P_{k}(z)=\sum_{j=0}^{m_{k}-1} a_{j, k} z^{j}
$$

Since $\left\|P_{k}\right\|_{2}^{2}=1, \sum_{j=0}^{m_{k}-1} a_{j, k}^{2}=1$. Write $p_{j, k}=a_{j, k}^{2}$.

We will now a construct a rank one non-singular transformation of generalized odometer type, equivalently, a rank one transformation where no spacers added at any stage of construction. This is done by the method of cutting and stacking $\mathbf{9}$ as follows. Let $\Omega_{0}=\Omega_{0,0}$ denote the unit interval. At stage one of the construction we divide $\Omega_{0}$ into $m_{1}$ pairwise disjoint intervals, $\Omega_{0,1}, \Omega_{1,1} \cdots, \Omega_{m_{1}-1,1}$, of lengths $p_{0,1}, p_{1,1}, \cdots, p_{m_{1}-1,1}$ respectively. For each $j, 0 \leq j \leq m_{1}-2$, we map the interval 
$\Omega_{j, 1}$ linearly onto $\Omega_{j+1,1}$. We view the intervals $\Omega_{0,1}, \Omega_{1,1} \ldots, \Omega_{m_{1}-1,1}$ as stacked one above the other. We thus get a stack of certain height $h_{1}=m_{1}$, together with a map $T$ which is defined on all intervals of the stack except the interval $\Omega_{m_{1}-1,1}$ at the top of the stack. Note that since $p_{j, 1} \neq p_{j+1,1}, T$ will not be measure preserving. This completes the first stage of the construction.

Suppose we have obtained at the end of $(k-1)$ th stage of construction a stack of height $h_{k-1}=m_{1} m_{2} \cdots m_{k-1}$ and a map $T$ which maps each interval of the stack linearly onto one above it, except that $T$ remains undefined on the top piece of the stack. Let $\Omega_{0, k-1}$ denote the bottom interval of this stack. $T^{h_{k-1}-1} \Omega_{0, k-1}$ is then the top of the stack. At $k$-th stage of construction we divide $\Omega_{0, k-1}$ into $m_{k}$ intervals $\Omega_{0, k}, \Omega_{1, k}, \cdots, \Omega_{m_{k}-1, k}$ in the ratios $p_{0, k}, p_{1, k}, \cdots, p_{m_{k}-1, k}$, and map, for $0 \leq j \leq m_{k}-2$, the interval $T^{h_{k-1}-1} \Omega_{j, k}$ linearly onto $\Omega_{j+1, k}$. We thus get, at the end of $k$-th stage of construction a stack of height $h_{k}=m_{1} m_{2} \cdots m_{k}$, where each piece of the stack is mapped by $T$ linearly onto the one above it except that $T$ remains undefined on the top piece of the stack which is $T^{h_{k-1}-1} \Omega_{m_{k}-1, k}$. Since $p_{i, k}$ 's are bounded away from 1 , we see that measure of $T^{h_{k-1}-1} \Omega_{m_{k}-1, k}$ goes to 0 as $k$ tends to $\infty$, whence $T$ is eventually defined almost everywhere and we get a non-singular transformation $T$ on $[0,1]$ which we call non-singular generalized odometer. Since $p_{k, j}$ 's are bounded away from 0 over all $k, j$, the maximum length of the intervals of $k$-th stack goes to 0 as $k$ tends to $\infty$. So a Lebesgue density argument allows us to prove that $T$ is ergodic. We omit this proof.

We also remark that there is no finite measure on Borel subsets of $[0,1]$ which is invariant under $T$ and mutually absolutely continuous with respect to $\nu$. This in turn implies that the unitary operators $U_{T}$ and $V_{\phi}$ defined in the next section do not admit eigenvalues.

The action $f \rightarrow f \circ T$ does admit a countable dense subgroup of $S^{1}$ as eigenvalues with measurable eigenfunctions of absolute value 1 and which separate points $[0,1]$ module $\nu$-null sets. Let $\Gamma$ denote this group of eigenvalues of $T$. For each

$\gamma \in \Gamma$ we can choose an eigenfunction $e_{\gamma}$ which is measurable, of absolute value 1 , and satisfies for all $\gamma, \gamma_{2} \in \Gamma, e_{\gamma_{1} \gamma_{2}}=e_{\gamma_{1}} e_{\gamma_{2}}$ modulo $\nu$ null sets. We will use these facts in the next section to prove that the maximal spectral type of $U_{T}$ is quasi-invariant and ergodic under translation action of $\Gamma$ on $S^{1}$.

\section{5. $V_{\phi}$, Lebesgue nature of its Spectrum}

Let $\phi$ denote a function on $[0,1]$, constant on each level of any stack except the top level, assuming values -1 and 1 . This function will be constructed inductively as we proceed. 
Let $T, \phi$ be as above. On $L^{2}([0,1], \nu)$ define

$$
\begin{gathered}
\left(U_{T} f\right)(x) \stackrel{\text { def }}{=}\left(\frac{d \nu \circ T}{d \nu}(x)\right)^{1 / 2} f(T x), f \in L^{2}([0,1], \nu), \\
\left(V_{\phi} f\right)(x) \stackrel{\text { def }}{=}(V f)(x)=\phi(x) \cdot\left(U_{T} f\right)(x), f \in L^{2}([0,1], \nu) .
\end{gathered}
$$

$U_{T}$, and $V$ are unitary operators. Since one has the formula

and more generally for $n \geq 0$

$$
\frac{d \nu \circ T^{2}}{d \nu}=\frac{d \nu \circ T}{d \nu} \frac{d \nu \circ T}{d \nu} \circ T
$$

$$
\frac{d \nu \circ T^{n}}{d \nu}=\frac{d \nu \circ T}{d \nu} \frac{d \nu \circ T^{n-1}}{d \nu} \circ T
$$

We have for $n \geq 0$,

$$
\begin{gathered}
\left(U_{T}^{n} f\right)(x)=\left(\frac{d \nu \circ T^{n}}{d \nu}(x)\right)^{1 / 2} f\left(T^{n} x\right), \\
\left(V^{n} f\right)(x)=\left(\prod_{j=0}^{n-1} \phi\left(T^{j}(x)\right)\right)\left(\frac{d \nu \circ T^{n}}{d \nu}(x)\right)^{1 / 2} f\left(T^{n} x\right),
\end{gathered}
$$

Since any bounded measurable function can be approximated in $L^{2}([0,1], \nu)$ by finite linear combinations of $V^{j} 1_{\Omega_{0, n}}, 0 \leq j \leq h_{n}-2$ for large enough $n, V$ has multiplicity one.

Write $T f=f \circ T$. Using facts such as $(A B)^{-1}=B^{-1} A^{-1}$ for invertible operators $A, B$, and that multiplication operators commute, we have from the formula for $V^{n} f$ given above:

$$
\begin{gathered}
\left(V^{-n} f\right)(\cdot)=T^{-n} \circ\left(\left(\prod_{j=0}^{n-1} \phi\left(T^{j}(\cdot)\right)\right)^{-1}\left(\frac{d\left(\nu \circ T^{n}\right)}{d \nu}(\cdot)\right)^{-1 / 2} f(\cdot)\right) \\
=\left(\prod_{j=0}^{n-1} \phi\left(T^{j-n}(\cdot)\right)\right)^{-1}\left(\frac{d \nu \circ T^{n}}{d \nu}\left(T^{-n}(\cdot)\right)\right)^{-1 / 2} f\left(T^{-n}(\cdot)\right)
\end{gathered}
$$

whence

$$
\left(T^{-n} f\right)(\cdot)=\prod_{j=0}^{n-1} \phi\left(T^{j-n}(\cdot)\right)\left(\frac{d\left(\nu \circ T^{n}\right)}{d \nu}\right)^{1 / 2}\left(T^{-n}(\cdot)\right)\left(V^{-n} f\right)(\cdot) .
$$

Recall that $\Omega_{0, k-1}$ is the base of the stack of height $h_{k-1}$ and $\Omega_{0, k}, \Omega_{1, k}, \cdots, \Omega_{m_{k}-1, k}$ is a partition of $\Omega_{0, k-1}$. We have

$$
\Omega_{0, k-1}=\bigcup_{j=0}^{m_{k}-1} T^{R_{j, k}}\left(\Omega_{0, k}\right)
$$


where $R_{j, k}=j h_{k-1}$ is the $j$-th return time of a point in $\Omega_{0, k}$ into $\Omega_{0, k-1}$.

$$
\begin{aligned}
1_{\Omega_{0, k-1}} & =\sum_{j=0}^{m_{k}-1} 1_{\Omega_{0, k}} \circ T^{-R_{j, k}} \\
& =1_{\Omega_{0, k}}+\sum_{j=1}^{m_{k}-1} c_{j, k}\left(\frac{d \nu \circ T^{R_{j, k}}}{d \nu} \circ T^{-R_{j, k}}\right)^{1 / 2}(\cdot)\left(V^{-R_{j, k}} 1_{\Omega_{0, k}}\right)(\cdot) \\
& =W_{k}(V) 1_{\Omega_{0, k}},
\end{aligned}
$$

where

$$
\left.W_{k}(z)=1+\sum_{j=1}^{m_{k}-1} c_{j, k}\left(\frac{d \nu \circ T^{R_{j, k}}}{d \nu}\left(T^{-R_{j, k}}\right)\right)\right)^{1 / 2}(\cdot)\left(z^{-R_{j, k}}\right),
$$

and $c_{j, k}=\prod_{i=0}^{R_{j, k}-1} \phi\left(T^{i-R_{j, k}}(x)\right), x \in \Omega_{j, k}, 1 \leq j \leq m_{k}-1$, a constant of absolute value one. Note that the constants $c_{j, k}$ 's can be preassigned and $\phi$ can be so defined that the above relation holds for all $(j, k)$. We will define $\phi$ inductively in such a way that $c_{j, k}=-1$, for all $j, k, 1 \leq j \leq m_{k}-1$. This will ensure, after we have substituted values of the Radon-Nikodym derivatives, that

$$
W_{k}(z)=-\frac{1}{\alpha} P_{k}\left(z^{h_{k-1}}\right) .
$$

Choose $\phi=-1$ on $\Omega_{0,1}$ and equal to 1 on $\Omega_{j, 1}, 1 \leq j \leq m_{1}-2$, and verify that

$$
c_{j, 1}=\prod_{i=0}^{R_{j, 1}-1} \phi\left(T^{i-R_{j, 1}} x\right)=-1, x \in \Omega_{j, 1}, 1 \leq j \leq m_{1}-1 .
$$

Assume now that $\phi$ has been defined on first $h_{k-1}-1$ levels of the stack of height $h_{k-1}, \phi$ is constant on each level with value -1 or 1 , such that for $l \leq k-1$

$$
c_{j, l}=-1,1 \leq j \leq m_{l}-1 .
$$

Let $b=\prod_{i=0}^{h_{k-1}-2} \phi\left(T^{i}(x)\right), x \in \Omega_{0, k-1}$, a constant independent of $x \in \Omega_{0, k-1}$, of value -1 or 1 . We now define $\phi$ on the intervals $T^{h_{k-1}-1} \Omega_{j, k}, 0 \leq j \leq m_{k}-2$ as follows: if $b=1$ the value of $\phi$ is -1 on $T^{h_{k-1}-1} \Omega_{0, k}$, and 1 on the intervals $T^{h_{k-1}-1} \Omega_{j, k}, 1 \leq j \leq m_{k}-2$. if $b=-1$ the value of $\phi$ is 1 on $T^{h_{k-1}-1} \Omega_{0, k}$, and -1 on the intervals $T^{h_{k-1}-1} \Omega_{j, k}, 1 \leq j \leq m_{k}-2$. This ensures that $c_{j, k}=-1$, $1 \leq j \leq m_{k}-2$. Thus we have defined $\phi$ inductively on all of $[0,1]$ so that $c_{j, k}=-1$, $1 \leq j \leq m_{k}-1, k=1,2 \cdots$.

We now observe that for $x \notin T^{R_{j, k}} \Omega_{0, k}$,

$$
V^{-R_{j, k}} 1_{\Omega_{0, k}}(x)=0,
$$

and that for $x \in T^{R_{j, k}} \Omega_{0, k}$,

$$
\frac{d \nu \circ T^{R_{j, k}}}{d \nu}\left(T^{-R_{j, k}}(x)\right)=\frac{p_{j, k}}{p_{0, k}} .
$$


We thus have, with $c_{0, k}=1, R_{0, k}=0$,

$$
1_{\Omega_{0, k-1}}=\sum_{j=0}^{m_{k}-1} c_{j, k}\left(\frac{p_{j, k}}{p_{0, k}}\right)^{1 / 2}\left(V^{-R_{j, k}} 1_{\Omega_{0, k}}\right)(\cdot) .
$$

Let us normalize $1_{\Omega_{0, k}}$ and write

$$
\begin{gathered}
f_{k}=\left(\frac{1}{\nu\left(\Omega_{0, k}\right)}\right)^{1 / 2} 1_{\Omega_{0, k}}=\left(\frac{1}{\prod_{j=1}^{k} p_{0, j}}\right)^{1 / 2} 1_{\Omega_{0, k}}, \\
f_{k-1}=\left(p_{0, k}\right)^{1 / 2}\left(1+c_{1, k}\left(\frac{p_{1, k}}{p_{0, k}}\right)^{1 / 2} V^{-R_{1, k}}+\cdots+c_{m_{k}-1, k}\left(\frac{p_{m_{k}-1, k}}{p_{0, k}}\right)^{1 / 2} V^{-R_{m_{k}-1, k}}\right) f_{k} .
\end{gathered}
$$

Now $\nu\left(\Omega_{0,0}\right)=1$ so $f_{0}=1_{\Omega_{0,0}}$. We have by iteration

$$
f_{0}=\left(\prod_{j=1}^{k} S_{j}(V)\right) f_{k}
$$

where

$S_{j}(z)=\left(p_{0, j}\right)^{1 / 2}\left(1+c_{1, j}\left(\frac{p_{1, j}}{p_{0, j}}\right)^{1 / 2} z^{-R_{1, j}}+\cdots+c_{m_{j}-1, j}\left(\frac{p_{m_{j}-1, j}}{p_{0, j}}\right)^{1 / 2} z^{-R_{m_{j}-1, j}}\right)$.

Note that by choice of $p_{i, j}$ and $c_{i, j}$,

$$
S_{j}(z)=-P_{j}\left(z^{h_{j-1}}\right), j=1,2, \cdots,\left|S_{j}(z)\right|^{2}=\left|P_{j}\left(z^{h_{j-1}}\right)\right|^{2} .
$$

Let $V^{n}=\int_{S^{1}} z^{-n} d E, n \in \mathbb{Z}$, be the spectral resolution of the unitary group $V^{n}, n \in \mathbb{Z}$, and

$$
\left(V^{n} f_{k}, f_{k}\right)=\int_{S^{1}} z^{-n}\left(E(d z) f_{k}, f_{k}\right)=\int_{S^{1}} z^{-n} d \sigma_{k}
$$

where $\sigma_{k}(\cdot)=\left(E(\cdot) f_{k}, f_{k}\right)$; the maximal spectral type of $E$ is given by $\vee_{k=0}^{\infty} \sigma_{k}$.

Since $T^{i} \Omega_{0, k}, 0 \leq i \leq h_{k}-1$ are pairwise disjoint, we see that the sequence $\sigma_{k}, k=1,2, \cdots$ converges weakly to $d z$ as $k \rightarrow \infty$.

We have for all integers $l$

$$
\left(V^{l} f_{0}, f_{0}\right)=\int_{S^{1}} z^{-l} d \sigma_{0}=\int_{S^{1}} z^{-l} \prod_{j=0}^{k}\left|P_{j}\left(z^{h_{j-1}}\right)\right|^{2} d \sigma_{k},
$$

whence

$$
d \sigma_{0}=\prod_{j=1}^{k}\left|P_{j}\left(z^{h_{j-1}}\right)\right|^{2} d \sigma_{k} .
$$

Since the spectral measure $E$ is atomfree and $P_{j}, 1 \leq j \leq k$, can vanish only at finitely points we see that for all $k, \sigma_{0}$ and $\sigma_{k}$ are mutually absolutely continuous, whence $\sigma_{0}$ is the maximal spectral type of $V_{\phi}$. 
Now $\sigma_{k} \rightarrow d z$ weakly as $k \rightarrow \infty$ and $\prod_{j=1}^{k}\left|P_{j}\left(z^{h_{j-1}}\right)\right|^{2} \rightarrow g^{2}$ uniformly, whence $\prod_{j=1}^{k}\left|P_{j}\left(z^{h_{j-1}}\right)\right|^{2} d \sigma_{k} \rightarrow g^{2} d z$ weakly as $k \rightarrow \infty$. Thus $\sigma_{0}$ is the measure $g^{2} d z$. Thus the maximal spectral type of $V_{\phi}$ is mutually absolutely continuous with respect to the Lebesgue measure.

We will now show that the maximal spectral type of $U_{T}$ is quasi-invariant and ergodic with respect to the translation action by $\Gamma$ on $S^{1}$. Consider the unitary group $J_{\gamma}: f \rightarrow e_{\gamma} f, f \in L^{2}([0,1], \nu), \gamma \in \Gamma$, which satisfies with $U_{T}$ the Weyl commutation relation $U_{T} J_{\gamma}=\gamma J_{\gamma} U_{T}$. Since the functions $e_{\gamma}, \gamma \in \Gamma$, separate points and $T$ is ergodic with respect to $\nu$ the pair of unitary groups $\left(U_{T}^{n}, n \in \mathbb{Z}, J_{\gamma}, \gamma \in \Gamma\right)$, is irreducible in the sense that only closed subspaces of $L^{2}([0,1], \nu)$ invariant under all $U_{T}^{n}, n \in \mathbb{Z}$, and all $J_{\gamma}, \gamma \in \Gamma$, are the trivial ones. This in turn implies that the spectral measure $F$ of $U_{T}$ satisfies with $J_{\gamma}, \gamma \in \Gamma$, the relation $J_{\gamma} F(\cdot) J_{\gamma^{-1}}=F((\cdot) \gamma), \gamma \in \Gamma$, and the system $\left(F, J_{\gamma}, \gamma \in \Gamma\right)$ is irreducible. It follows from this that the maximal spectral type of $F$, say $\sigma$, is ergodic and quasi-invariant under $\Gamma$. Since $\Gamma$ is dense in $S^{1}$ and $\sigma$ is ergodic under $\Gamma$ we see that $\sigma$ is either equivalent to $d z$ on $S^{1}$ or singular to it. (17, 11.11, 12.14,13.3, $13.4,13.5)$.

Next we show that the maximal spectral type of $U_{T}$ is singular to $(d z)$. Recall that the constant term of $a_{0, j}$ of $P_{j}(z)$ is negative and converges to $-\alpha$ as $j \rightarrow \infty$ and that $P_{j}(z) \rightarrow \frac{z-\alpha}{1-\alpha z}$ as $j \rightarrow \infty$. The polynomial $L_{j}(z) \stackrel{\text { def }}{=} 2\left|a_{0, j}\right|+P_{j}(z)$ have $L_{2}\left(S^{1}, d z\right)$ norm 1 and

$$
\left|L_{j}(z)\right| \rightarrow\left|\frac{z-\alpha}{1-\alpha z}+2 \alpha\right|
$$

uniformly as $j \rightarrow \infty$. The function $\left|\frac{z-\alpha}{1-\alpha z}+2 \alpha\right|$ is non-constant, it has $L^{2}\left(S^{1}, d z\right)$ norm 1 since each $L_{j}$ has $L^{2}\left(S^{1}, d z\right)$ norm 1 . So its $L^{1}\left(S^{1}, d z\right)$, norm, say $l$, is strictly less than one, whence, as $j \rightarrow \infty, \int_{S^{1}}\left|L_{j}(z)\right| d z \rightarrow l<1$.

Lemma. If the maximal spectral type of $U_{T}$ has the same null sets as $d z$ then $\int_{S^{1}}\left|L_{n}(z)\right| d z \rightarrow 1$ as $n \rightarrow \infty$.

(Note that this contradicts what is stated just above, so the maximal spectral type of $U_{T}$ is singular to $d z$ by the dichotomy result.)

Proof. The maximal spectral type of $U_{T}$ is given by the weak limit of measures $\prod_{j=1}^{n}\left|L_{j}\left(z^{h_{j-1}}\right)\right|^{2} d z, n=1,2, \cdots$. It is known that $\prod_{j=1}^{n}\left|L_{j}\left(z^{h_{j-1}}\right)\right|, n=1,2, \cdots$ converge over a subsequence, say $n_{k}, k=1,2, \cdots$, to $\sqrt{\frac{d \sigma}{d z}}$ a.e. $(d z)$, (see [1], Proposition 5.2). Assume that $\sigma$ and $d z$ have the same null sets. Then there is a 
subsequence $n_{k}, k=1,2, \cdots$ such that, for almost all $z$, we have

$$
\prod_{j=1}^{n_{k}}\left|L_{j}\left(z^{h_{j-1}}\right)\right|(z) \underset{k \rightarrow+\infty}{\longrightarrow} \sqrt{\frac{d \sigma}{d z}}(z)
$$

Since $\frac{d \sigma}{d z}(z)$ is positive a.e. $(d z)$ the partial products $\prod_{j=n_{k}+1}^{n_{k+1}} \mid L_{j}\left(z^{h_{j-1}} \mid, k=1,2, \cdots\right.$ converge almost everywhere $(d z)$ to 1 . We thus get,

$$
\int_{S^{1}} \prod_{j=n_{k}+1}^{n_{k+1}}\left|L_{j}\left(z^{h_{j-1}}\right)\right| d z \underset{k \rightarrow+\infty}{\longrightarrow} 1
$$

since, by Cauchy-Schwarz inequality, for all $k$,

$$
\int_{S^{1}} \prod_{j=n_{k}+1}^{n_{k+1}}\left|L_{j}\left(z^{h_{j-1}}\right)\right| d z \leq 1 .
$$

Let $M_{k}$ be any $L_{j}, n_{k}+1 \leq j \leq n_{k+1}$. We will show that

$$
\int_{S^{1}} \prod_{j=n_{k}+1}^{n_{k+1}}\left|L_{j}\left(z^{h_{j-1}}\right)\right| d z \leq\left(\int_{S^{1}}\left|M_{k}(z)\right| d z\right)^{\frac{1}{2}}
$$

wherein letting $k \rightarrow \infty$ the Lemma follows. To fix ideas we take $M_{k}=L_{n_{k+1}}$. Now, by Cauchy-Schwarz inequality, we have

$$
\begin{aligned}
& \int_{S^{1}} \prod_{j=n_{k}+1}^{n_{k+1}}\left|L_{j}\left(z^{h_{j-1}}\right)\right| d z=\int_{S^{1}} \sqrt{\left|L_{n_{k+1}}\left(z^{h_{n_{k+1}}-1}\right)\right|} \sqrt{\left|L_{n_{k+1}}\left(z^{h_{n_{k+1}-1}}\right)\right|} \prod_{j=n_{k}+1}^{n_{k+1}-1}\left|L_{j}\left(z^{h_{j-1}}\right)\right| d z \\
& \leq\left(\int_{S^{1}}\left|L_{n_{k+1}}\left(z^{h_{n_{k+1}-1}}\right)\right| d z\right)^{\frac{1}{2}}\left(\int_{S^{1}}\left|L_{n_{k+1}}\left(z^{h_{n_{k+1}-1}}\right)\right| \prod_{n_{k}+1}^{n_{k+1}-1}\left|L_{j}\left(z^{h_{j-1}}\right)\right|^{2} d z\right)^{\frac{1}{2}} \\
& \leq\left(\int_{S^{1}}\left|L_{n_{k+1}}\left(z^{h_{n_{k+1}-1}}\right)\right| d z\right)^{\frac{1}{2}},
\end{aligned}
$$

since, again by Cauchy-Schwarz inequality, we have

$$
\begin{gathered}
\int_{S^{1}}\left|L_{n_{k+1}}\left(z^{h_{n_{k+1}}-1}\right)\right| \prod_{j=n_{k}+1}^{n_{k+1}-1}\left|L_{j}\left(z^{h_{j-1}}\right)\right|^{2} d z \\
=\int_{S^{1}} \prod_{j=n_{k}+1}^{n_{k+1}}\left|L_{j}\left(z^{h_{j-1}}\right)\right| \prod_{j=n_{k}+1}^{n_{k+1}-1}\left|L_{j}\left(z^{h_{j-1}}\right)\right| d z \\
\leq\left(\int_{S^{1}} \prod_{j=n_{k}+1}^{n_{k+1}}\left|P_{j}\left(z^{h_{j-1}}\right)\right|^{2} d z\right)^{\frac{1}{2}}\left(\int_{S^{1}}^{n_{k=n_{k}+1}} \prod_{j}^{n_{j}-1}\left|L_{j}\left(z^{h_{j-1}}\right)\right|^{2} d z\right)^{\frac{1}{2}}=1 .
\end{gathered}
$$

The lemma follows. 
We now construct the desired non-singular non-dissipative ergodic transformation $\tau$ with Lebesgue component in the spectrum of $U_{\tau}$. Since $V_{\phi}$ has spectrum different from that of $U_{T}, \phi$ is not a multiplicative coboundary, i.e, it is not of the form $\frac{\xi \circ T}{\xi}$, for any measurable function $\xi$. Consider now the space $X=[0,1] \times\{-1,1\}$, equipped with product measure, denoted by $\mu$, where $[0,1]$ has Lebesgue measure and $\{-1,1\}$ has uniform probability distribution. View $\{-1,1\}$ as a multiplicative group with two character $\xi_{0}, \xi_{1}$, with $\xi_{0}$ the identity character. Define $\tau(x, y)=(T x, \phi(x) y),(x, y) \in X$. The transformation $\tau$ is non-singular with respect to $\mu$, and ergodic since $\phi$ is not a multiplicative coboundary. Define

$$
U_{\tau} f=\sqrt{\frac{d \mu \circ \tau}{d \mu}} f \circ \tau, f \in L^{2}(X, \mu) .
$$

The space $L^{2}(X, \mu)$ is orthogonal sum of $L^{2}([0,1], \nu)$ and $\xi_{1} L^{2}([0,1], \nu)$ each invariant under $U_{\tau}$. The restriction of $U_{\tau}$ to $L^{2}([0,1], \nu)$ is $U_{T}$ and its restriction to $\xi_{1} L^{2}([0,1], \nu)$ is $V_{\phi}$ since $\xi_{1}(\phi)=\phi$. Clearly $U_{\tau}$ admits Lebesgue component of multiplicity one.

\section{Remarks}

1) There is no ultraflat sequence of polynomials with non-negative coefficients which are bounded away from one. For, if $P_{n}(z)=\sum_{j=0}^{m_{n}} a_{j, n} z^{j}, n=1,2, \cdots$, is an ultraflat sequence of polynomials such that for each $n, 0 \leq a_{j, n}<\lambda<1$, $0 \leq j \leq m_{n}$ then since it is ultraflat

$$
\sum_{j=0}^{m_{n}} a_{j, n}^{2}=1<\sum_{j=1}^{m_{n}} a_{j, n}=P_{n}(1),
$$

whence

$$
P_{n}(1)-1=\sum_{j=0}^{m_{n}}\left(a_{j, n}-a_{j, n}^{2}\right)>1-\lambda,
$$

contradicting that $P_{n}(1) \rightarrow 1$ as $n \rightarrow \infty$.

2) We do not know if there exists a sequence $P_{n}, n=1,2, \cdots$ of analytic polynomials of $L^{2}\left(S^{1}, d z\right)$ norm 1 , with coefficients non-negative and bounded away from 1 , such that $\left|P_{n}(z)\right| \rightarrow 1$ a.e $(d z)$ as $n \rightarrow \infty$. An affirmative answer will imply that there are ergodic, non-dissipative, non-singular transformations $\tau$ with simple Lebesgue spectrum for $U_{\tau}$, answering a question of Banach in the non-singular category [1], 2], 6]. In particular it is not known if for a nondissipative non-singular generalized odometer action $T, U_{T}$ always has singular spectrum. Using the Peyrière-Brown method [18, [5], it is can be shown that 
the spectrum of the generalized odometer is singular if the following holds

$$
\sum_{k \geq 1} \frac{\left|\sum_{i \neq j} \sqrt{p_{i, k} p_{j, k}}\right|^{2}}{2\left(m_{k}-1\right)}=+\infty .
$$

3) The Banach problem asks if there is a Lebesgue measure preserving transformation on $\mathbb{R}$ which has simple Lebesgue spectrum. This problem is stated in 1959's Ulam book [21, p.76]. A similar problem is mentioned by Rokhlin in [20]. Precisely, Rokhlin asked on the existence of an ergodic measure preserving transformation on a finite measure space whose spectrum is Lebesgue type with finite multiplicity. Later, in 1966, Kirillov in $\mathbf{1 4}$ wrote "there are grounds for thinking that such examples do not exist". However he has described a measure preserving action (due to M. Novodvorskii) of the group $\left(\bigoplus_{j=1}^{\infty} \mathbb{Z}\right) \times\{-1,1\}$ on the compact dual of discrete rationals whose unitary group has Haar spectrum of multiplicity 2. Similar group actions with higher finite even multiplicities are also given.

4) Finite measure preserving $T$ with $U_{T}$ having Lebesgue component of finite even multiplicity have been constructed by J. Mathew and M. G. Nadkarni[16], M. Queffelec [19], and O. Ageev[3].

5) For the case of the non-singular maps, Ismagilov established the connection between the classical Riesz product and the spectral type of some Mackey actions [10, 11, 12. For all those examples, he proved that the spectrum is singular.

\section{References}

[1] E. H. el Abdalaoui and M. Nadkarni, Calculus of generalized Riesz products, preprint 2013, http://fr.arxiv.org/pdf/1307.6513

[2] E. H. el Abdalaoui and M. Nadkarni, Some notes on flat polynomials, preprint 2014, http://fr.arxiv.org/abs/1402.5457

[3] O Ageev, Dynamical system with an even-multiplicity Lebesgue component in the spectrum, Math. USSR, 64, 1987, 305.

[4] J. Bourgain, On the spectral type of Ornstein class one transformations, Israel J. Math., 84 (1993), 53-63.

[5] B. Brown, Singular infinitely divisible distributions whose characteristic functions vanish at infinity, Math. Proc. Cambridge Philos. Soc. 82 (1977), no. 2, 277-287.

[6] J. R. Choksi and M. G. Nadkarni, On the question of transformations with simple Lebesgue spectrum, Lie groups and ergodic theory (Mumbai, 1996), 33-57, Tata Inst. Fund. Res. Stud. Math., 14, Tata Inst. Fund. Res., Bombay, 1998.

[7] M. Guenais, Morse cocycles and simple Lebesgue spectrum, Ergodic Theory Dynam. Systems, 19 (1999), no. 2, 437-446.

[8] T.Downarowicz, Y. Lacroix, Merit factors and Morse sequences, Theoretical Computer Science, (209) (1998), 377-387.

[9] N. Friedman, Introduction to ergodic theory, van Nostrand-Reinhold, New York, 1970.

[10] R. S. Ismagilov, Riesz products, random walk, and spectrum, (Russian) Funktsional. Anal. i Prilozhen. 36 (2002), no. 1, 16-29, 96; translation in Funct. Anal. Appl. 36 (2002), no. 1, $13-24$

[11] R. S. Ismagilov, The spectrum of dynamical systems and the Riesz products, (Russian) Mat. Sb. 180 (1989), no. 7, 888-912, 991; translation in Math. USSR-Sb. 67 (1990), no. 2, 341-366 
[12] R. S. Ismagilov, Riesz products and the spectrum of the Mackey action, (Russian) Funktsional. Anal. i Prilozhen. 20 (1986), no. 3, 86-87.

[13] J-P. Kahane, Sur les polynômes à coefficients unimodulaires, (French) Bull. London Math. Soc., 12 (1980), no. 5, 321-342.

[14] A. A. Kirillov, Dynamical systems, factors and group representations, (Russian) Uspehi Mat. Nauk 221967 no. 5 (137), 67-80.

[15] Littlewood, J. E. (1966). On polynomials $\sum^{n} z^{m}, \sum_{m=0}^{n} e^{\alpha i} z^{m}, z=e^{\theta i}$, J. London Math. Soc. 41, 1966, 367-376.

[16] J. Mathew and M.G. Nadkarni,A measure-preserving transformation whose spectrum has Lebesgue component of multiplicity two, Bull. London Math. Soc 16 (1984), 402-406.

[17] M. G. Nadkarni, Spectral theory of dynamical systems, Hindustan Book Agency, New Delhi, (1998); Birkhäuser Advanced Texts : Basler LehrbÆcher. [Birkhäuser Advanced Texts: Basel Textbooks] Birkhäuser Verlag, Basel, 1998.

[18] J. Peyrière, Étude de quelques propriétés des produits de Riesz, Ann. Inst. Fourier, Grenoble, 252 (1975), 127-169.

[19] M. Queffélec, Une nouvelle propriété des suites de Rudin-Shapiro, Ann. Inst. Fourier 37 (1987), 115-138.

[20] V. A. Rokhlin, Selected topics in the metric theory of dynamical systems, Uspekhi Mat. Nauk "New series", 4 (1949), 57-128 (Russian); Amer. Math. Soc. Transl. 2, 40 (1966), 171-240.

[21] S. M. Ulam, Problems in modern mathematics, Science Editions John Wiley \& Sons, Inc., New York 1964.

Normandie University, University of Rouen Department of Mathematics, LMRS UMR 6085 CNRS, Avenue de L'Université, BP.12 76801 Saint Etienne du Rouvray FRANCE

E-mail address: elhoucein.elabdalaoui@univ-rouen.fr

URL: http://www.univ-rouen.fr/LMRS/Persopage/Elabdalaoui/

Department of Mathematics, University of Mumbai, Vidyanagari, Kalina, Mumbai, 400098, INDIA

E-mail address: mgnadkarni@gmail.com

URL: http://insaindia.org/detail.php?id=N91-1080 